\title{
Characterizing Multiplexing and Diversity in Visual MIMO
}

\author{
Ashwin Ashok ${ }^{\ddagger}$, Marco Gruteser ${ }^{\ddagger}$, Narayan Mandayam ${ }^{\ddagger}$, Kristin Dana $^{\dagger}$ \\ ${ }^{\ddagger}$ WINLAB, Rutgers University, 671 Route 1 South, North Brunswick, NJ, USA \\ ${ }^{\dagger}$ Department of ECE, Rutgers University, 94 Brett Road, Piscataway, NJ, USA \\ Email: ${ }^{\ddagger}\{$ aashok,gruteser,narayan $\} @$ winlab.rutgers.edu, ${ }^{\dagger}{ }^{k}$ dana@ @ece.rutgers.edu
}

\begin{abstract}
Mobile optical wireless has so far been limited to very short ranges for high data rate systems. It may be feasible to overcome the data rate limitations over large transmission range in optical wireless through camera receivers and light emitting transmitter arrays through a concept what we call "visual MIMO". In this concept multiple transmit elements of a light emitting array (LEA) are used as transmitters to communicate to the individual pixel elements of the camera which act as multiple receive elements to create the visual MIMO channel. Multiplexing information over parallel data channels albeit be very similar to RF MIMO in concept, the visual MIMO approach dramatically differs in its characterization. In visual MIMO since the received signal is essentially the image of the transmitting element, the perspective distortions in the visual channel dominate over some of the important properties of a RF wireless channel such as distance based attenuation and multipath fading. Some of the prominent perspective distortions include the reduction in the size of the image with distance and skew/rotation in the image due to angular view. Further lens blur (typically due to focus imperfection or jerks while capturing the image) can also significantly depreciate the image quality. In this paper we will detail how MIMO techniques such as multiplexing and diversity are characterized based on the effect of perspective distortions. Based our visual MIMO channel model we will derive the analytical channel capacity of the visual MIMO channel and using the same we illustrate the significance of parameters such as distance, viewing angle and blur in characterizing multiplexing and diversity in visual MIMO.
\end{abstract}

\section{INTRODUCTION}

High data rate mobile optical wireless communications, has so far been limited to very short transmission ranges of less than $10 \mathrm{~m}$ [3]. To achieve transmission ranges greater than a few tens of meters in optical wireless requires highly directional light beams with very narrow angle-of-view [2]. Optical wireless channels are characterized by large path loss and high background noise typically from sunlight or other ambient light sources in vicinity [16]. Further the low transmit power levels in optical channels (due to output power regulations in optical sources such as LEDs and LASERs) limit the signal-to-noise ratios in these channels and thus the transmission range.

In our recent work in [6], we have argued that it is now becoming feasible to achieve high data rates over large transmission ranges in mobile optical wireless communications using camera receivers through a concept what we call "visual MIMO". In this concept, optical transmissions by an array of light emitting devices are received by an array of photodetector (pixels) elements of a camera. The pixels in a camera can essentially be viewed as an array of highly directional receive elements. Such a structure allows allows reducing interference and noise from other light sources in the channel. Such a system offers a degree of freedom in selecting and combining a subset of the receiver elements that receive a strong signal from the transmitter and thus achieve large SNRs. This may be very similar to the antenna selection in RF-MIMO but will incur lesser overhead and non-complex processing at the camera receiver as the processing can be done in software using image processing and computer vision algorithms [6]. However, the tradeoffs in the visual MIMO system, are a limited receiver sampling frequency and strong line-of-sight (LOS) requirements. We already showed in [6] that using visual MIMO it is possible to achieve considerable data rates over large transmission ranges with just a single transmitting element. Using MIMO techniques such as "multiplexing" to send independent streams of bits using the multiple elements of the light transmitter array and recording over a group of camera pixels can further enhance the data rates. On the other hand the system could send the same information on all the transmit elements of the array and use diversity combining at the camera to achieve large transmission ranges due to the SNR gain. Though the multiplexing and diversity techniques are similar in concept to those in RF MIMO systems [11] the visual MIMO channel with very different characteristics attributes certain unique behavior to the MIMO gains in these systems.

In visual MIMO the perspective distortions in the visual channel dominate over some of the important properties of a RF wireless channel such as distance based attenuation and multipath fading. Though perspective distortions in visual channels are primarily distance dependent visual MIMO channels induce perspective distortions in the image even if the transmitter and receiver are aligned at an angle with respect to each other. Two images which are clearly separated in the image plane may look overlapped when viewed from an angle. Such distortions can depreciate the signal quality and the detection capability leading to errors and thus reduction in the data rates. Further lens blur (typically due to focus imperfection or jerks while capturing the image) also can significantly depreciate the image quality and thus reduce the information capacity.

In this paper we will detail how MIMO techniques such 
as multiplexing and diversity are characterized based on the effect of perspective distortions in the visual MIMO channel. Based our channel model we will derive the analytical channel capacity of the visual MIMO channel and using the same we illustrate the significance of parameters such as distance, viewing angle and blur in characterizing multiplexing and diversity in visual MIMO.

This paper is structured as follows; in section III we detail the visual MIMO channel model followed by the perspective dependent MIMO characterizations in section IV-C. In section $\mathrm{V}$ we plot the analytical channel capacity in visual MIMO and follow up with key inferences about the multiplexing and diversity characterization in visual MIMO based on the capacity plots.

\section{RELATED WORK}

Prior work in optical wireless using visible light that use photodiode receivers or imaging receivers are either limited to short ranges or require complex processing at the receiver [17], [21], [22]. Though photo diodes can convert pulses at very high rates, they suffer from large interference and background light noise. This results in very low SNRs and thus short communication ranges. We showed analytically in [6], based on the visual MIMO concept, that a camera receiver outperforms photodiode receivers in terms of its channel capacity at medium to long ranges. Recently, a few sporadic projects have begun to investigate cameras as receivers, particularly for inter-vehicle communications [21] and traffic light to vehicle communications [8]. Their analytical results show that communication distances of about $100 \mathrm{~m}$ with a BER $\leq 10^{-6}$ are possible. Other work has investigated channel modeling [18] and multiplexing [7]. While earlier work has also used cameras to assist in steering of FSO transceivers [25], the visual MIMO approach differs by directly using cameras as receiver to design an adaptive visual MIMO system that uses multiplexing at short distances but still can achieve ranges of hundreds of meters in a diversity mode.

Only a few projects till now have investigated MIMO techniques for optical wireless. For shorter range systems [15], [26] show a MIMO approach for indoor optical wireless communication, [13] studied the capacity of a optical MIMO system and [19] details some work on space-time codes for optical MIMO. Earlier work by Kahn [23] investigates the use of multibeam transmitters and imaging receivers in Infra-Red systems very similar to MIMO in concept. Very recently the PixNet project [20] presents an implementation of an LCD - camera communication system that can deliver high data rates of the order of Mbps over distances of about $16 \mathrm{~m}$ and wide view angles. PixNet uses OFDM to transmit between the LCD-camera pair similar to the pixelated - MIMO system proposed by Hranilovic and Kschischang [13]. In this paper we will emphasize that regardless of any type of modulation and transmission scheme, visual MIMO can still achieve significantly high data rates by exploiting some of the unique characteristics of the visual channel.

\section{VISUAL MIMO MODEL}

In the visual MIMO communications system, the optical transmit element generates a light beam (optical signal) whose output power is proportional to the electrical input power of the modulating signal, limited by the emitter's peak transmission power [14], [18], [22]. While RF channels are typically characterized by their impulse response that reflects the multipath environment, this aspect differs significantly for optical channels. Since the rate of change of the channel impulse response is very slow compared to the frequency of the optical signal, it is usually sufficient to use a static parameter (channel DC gain) [16] to represent the channel. For the same reason inter-symbol interference and multipath fading can be neglected in optical wireless channels. Similarly Doppler shift is negligible compared to the frequency as well.

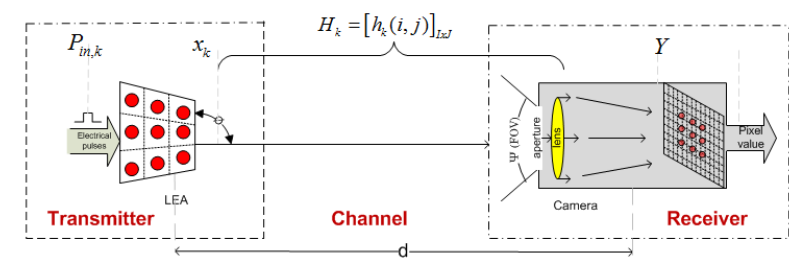

Fig. 1. The LEA-Camera visual MIMO communication model

Consider the visual MIMO communication system model as shown in Fig. 1 where an optical transmitter consisting of an array of $K$ transmitting elements communicates to a camera receiver with an array of $I \times J$ pixels. The channel model for the visual MIMO system is given as,

$$
\mathbf{Y}=\sum_{k=1}^{K} \mathbf{H}_{k} x_{k}+\mathbf{N}
$$

where $\mathbf{Y} \in \mathcal{R}^{I \times J}$ is the image current matrix with each element representing the received current $y(i, j)$ in each pixel with image coordinates $(i, j), x_{k} \in \mathcal{R}$ represents the transmitted optical power from $k^{\text {th }}$ element of the LEA and $\mathbf{H}_{k} \in \mathcal{R}^{I \times J}$ is the channel matrix of the $k^{t h}$ transmit element of the LEA, with elements $h_{k}(i, j)$ representing the channel between the $k^{t h}$ transmit element and pixel $(i, j)$, and $\mathbf{N}$ is the noise matrix. Noise in optical wireless is dominated by shot noise from background light sources and typically modeled as AWGN [16], [18]. Each element $n(i, j)$ of the noise matrix $\mathbf{N}$ representing the noise current at each pixel is given as,

$$
n(i, j)=\sqrt{\sigma_{s h o t}}=\sqrt{2 q R P_{n} s^{2} W}
$$

where $q$ is the electron charge, $R$ is the responsitivity of the receiver characterized as the optical power to current conversion factor, $P_{n}$ is the background shot noise power per unit area, $s$ is the square pixel side length and $W$ is the sampling rate of the receiver (equates to the frame rate of the camera).

The optical signal from the $k^{t h}$ transmit element $(k=$ $1,2,3 \ldots K)$ emitting a light beam of power $P_{i n, k}$ will be 
transmitted into the channel. At the receiver, depending on the focusing of the camera and the distance between the transmitting element and the camera, the transmitting element's image may strike a pixel or a group of pixels of the detector array. The signal current in each pixel will depend on the concentration of the received signal component on that pixel which can be quantified as the ratio of the pixel area relative to the area spanned by the transmitting element's image on the detector. If $c_{k}(i, j)$ represents the concentration ratio of the $k^{\text {th }}$ transmit element of an LEA on pixel $(i, j)$, the channel DC gain $h_{k}(i, j)$ from each transmit element $k$ to the pixel $(i, j)$ is given as

$h_{k}(i, j)=R \times R_{o}(\Phi) \times A_{\text {lens }} \times \frac{\cos (\psi) \cos ^{2}\left(\phi_{k, i, j}\right)}{d_{k, i, j}^{2}} \times c_{k}(i, j)$

where $R$ is the responsitivity, $R_{o}(\Phi)$ is the Lambertian radiation pattern of the optical transmitting element [16] with half-power angle $\Phi, A_{\text {lens }}$ is the area of the camera lens, $\psi$ is the camera field-of-view (fov) and $d_{k, i, j}, \phi_{k, i, j}$ are the distance \& viewing angle between each transmit element $k$ and receiving pixel $(i, j)$ respectively.

Typically, since the pixel size is very small (order of microns), the difference in distance $d_{k, i, j}$ and the viewing angle $\phi_{k, i, j}$ between each element of the transmitter array and every pixel is negligible. Therefore we refer to the distance $d_{k, i, j}=d$ and the viewing angle $\phi_{k, i, j}=\phi$ as the perpendicular distance and the angle between the transmitter array and image detector planes respectively. Hence the channel between each transmit element $k$ and each pixel $(i, j)$, characterized by $h_{k}(i, j)$, is primarily dependent on the concentration ratio $c_{k}(i, j)$ which can expressed as,

$$
\begin{gathered}
c_{k}(i, j)=\frac{s^{2}}{\pi\left(\frac{f l_{k}}{d}+\sigma_{\text {blur }}\right)^{2} / 4} \mathcal{I}_{k}(i, j) \\
\mathcal{I}_{k}(i, j)= \begin{cases}1 & \forall\left(i-i_{k}^{r e f}\right)^{2}+\left(j-j_{k}^{r e f}\right)^{2} \leq\left(\frac{f l_{k}}{d}+\sigma_{\text {blur }}\right)^{2} / 4 \\
0 & \text { otherwise }\end{cases}
\end{gathered}
$$

where, $s, f, l_{k}$ are the pixel edge length, camera focal length and diameter of $k^{\text {th }}$ transmit element (considering a circular transmitting element) respectively. The amount of concentration of the signal per pixel is also dependent on the amount of blur in the image due to the lens. Typically, lens blur is modeled as a Gaussian function [12] and the amount of blur in the image is quantified by its standard deviation $\left(\sigma_{\text {blur }}\right)$. The lens essentially acts like a filter with the blur function as its impulse response. Thus the image of the transmit element can be viewed as a result of the projected image convolving with the blur function over the detector area.

$\mathcal{I}($.$) is an indicator function indicating whether a pixel$ $(i, j)$ receives a signal from the transmit element $k$ or not, and is referenced in terms of the distance from pixel at the center of the transmit element's image $\left(i_{k}^{r e f}, j_{k}^{r e f}\right)$. Given the spatial coordinates of the transmitting elements of an LEA with respect to the camera reference we can determine the image center coordinates of those transmit element through optical ray-tracing techniques in conjunction with some basic computer vision theory [9].

\section{Perspective Dependent MiMo gains}

While the channel model in (1) resembles that of the familiar RF MIMO channel model, in fact it is significantly different from that. In RF MIMO systems, the channel matrix is typically a rich scattering matrix (usually full rank) whose entries are modeled well as independent and identically distributed random variables [10]. Further, this property allows the RF MIMO system to exploit either diversity and or multiplexing gains in data transmission which primarily depend on the multipath fading in the RF channel. The fact that the communication system here uses light as the communication medium, requires line of sight at the receiver, and the nature of the concentration function of the camera, renders some unique multiplexing and diversity characterizations different from RF MIMO.

\section{A. Resolvability of images}

The notion of 'parallel' channels to obtain the multiplexing data rate gains can be achieved only if the circumference of two transmit elements as seen on the image plane are separated by atleast a threshold $(\eta)$ number of pixels in both dimensions (horizontal and vertical). As we see in Fig. 2 even if the circumference of the two transmit element images are separated by one pixel they may not be resolvable because of the blur in the image. Hence we set a threshold distance of separation between the image circumferences, $\gamma=2 \sqrt{2 \ln 2} \sigma_{b l u r}$, equal to the full-width-half maximum (FWHM) of the Gaussian lens-blur function typically used as a parameter for image resolution in analyzing fine detailed astronomical and medical images [4], [5]. The distance of separation between the images of the transmit elements can be determined by perspective projection analysis (as described in [6]) considering circular transmitting elements. Given a fixed-focal length $f$ of the camera, pixel side length $s$ and a spatial distance $\alpha$ between the circumference of two adjacent LEDs, the circumference of two transmit element images will be separated by $\alpha_{i m}=\frac{f \alpha}{d s}$ pixels in each dimension. Therefore the separation between the circumference of two transmit element images will be equal to the threshold $(\gamma / s)$ at a distance $d^{*}=\frac{f \alpha}{\gamma}$ between the LEA and camera. This implies that, multiplexing in visual MIMO is possible only when $d \leq d^{*}$ and when $d>d^{*}$ each transmit element has to transmit the same information whereby diversity combining at the receiver can ensure an SNR gain and hence an equivalent capacity gain.

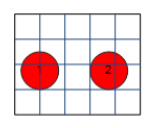

resolvable

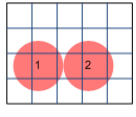

unresolvable
Fig. 2. Effect of lens blur on the resolvability of images 


\section{B. Dependence on viewing angle}

We can observe in equation (3) that the channel quality depreciates with the viewing angle $\phi$ (angle between the camera image plane and LEA surface plane). Two images which are clearly separated in the image plane may look overlapped when viewed from an angle. Such distortions can significantly depreciate the signal quality and the detection capability leading to errors and thus reduction in the data rates. Moreover such an angular view also reduces the achievable multiplexing transmission range. This is because when the camera image detector plane is at an angle $\phi$ to the transmitter array the effective spatial separation between two neighboring transmit elements becomes $\alpha \cos (\phi)(\leq \alpha)$ (as shown in Fig. 3). From the earlier discussion on the resolvability of images, it implies that the distance upto which multiplexing can be achieved in visual MIMO then reduces to

$$
d^{*}=\frac{f \alpha}{\gamma} \cos (\phi)
$$

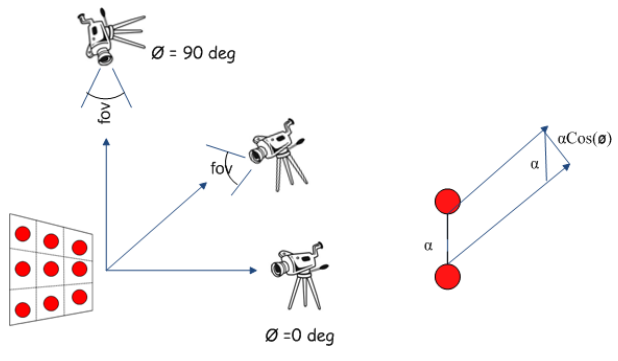

Fig. 3. Camera Viewing angle Illustration

\section{Distance dependent MIMO gains}

In the visual MIMO channel, for a static transmitter and receiver, the image of the LEA transmit elements captured by the camera spans one pixel or multiple pixels. Further, the image plane is spanned by images of each transmit element clearly delineated and the size of image span depending on the focus (concentration ratio) of the camera. As illustrated in Fig. 4, at short distances between the transmitter and receiver, each transmitting element of the LEA looks clearly focused on a unique set of pixels and the images of these elements can be detected from the complete image. In contrast, at a large distance between the transmitter and receiver, the image of each transmit element looks clearly unfocused and thus the signal from all the transmitting elements of the LEA is directed to typically one or few pixels. This suggests that at short distances, the system can offer large "multiplexing" gains by using the transmitting elements to signal independent bitstreams or equivalently realizing "parallel" channels. On the other hand, at large distances, there can only be a "diversity" gain where by the same bits are signaled on each of the transmit elements. These distance dependent gains in visual MIMO is in contrast to the RF MIMO channel, where the rich scattering channel matrix typically allows a continuous trade-off between diversity and multiplexing gains [24], [27].

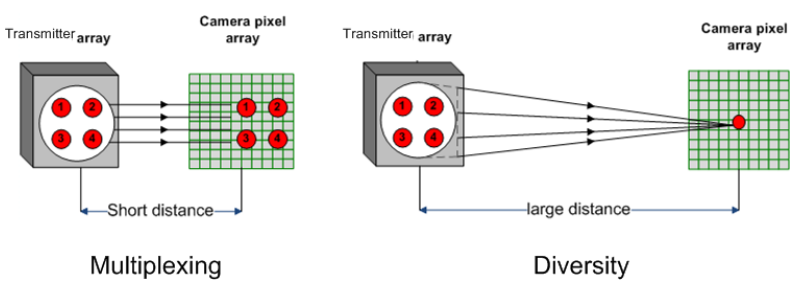

Fig. 4. Distance dependent Multiplexing and Diversity modes

\section{VISUAL MIMO CHANNEL CAPACITY}

To quantify the perspective dependent multiplexing and diversity gains in visual MIMO we use the channel capacity of the visual MIMO channel as a metric which is given as,

$$
\begin{gathered}
C=\left\{\begin{array}{l}
\sum_{k=1}^{K} W \log _{2}\left(1+S N R_{\text {cam }, k}\right) \quad \text { if } d \leq d^{*} \\
W \log _{2}\left(1+\sum_{k=1}^{K} S N R_{\text {cam }, k}\right) \quad \text { if } d>d^{*}
\end{array}\right. \\
S N R_{\text {cam }, k}=\frac{\sum_{\forall I_{k}(i, j)=1}\left(h_{k}(i, j) x_{k}\right)^{2}}{\sum_{\forall I_{k}(i, j)=1} n_{k}^{2}(i, j)}
\end{gathered}
$$

where $W$ is the receiver sampling rate (camera frame-rate), $d^{*}$ is the threshold multiplexing distance from equation (6). $S N R_{\text {cam }, k}$ is the signal-to-noise ratio of the $k^{t h}$ LED at the camera receiver [6] which is expressed in terms of the transmit power $x_{k}$, the channel DC gain $h_{k}(i, j)$ from equation (3) and AWGN noise $n_{k}(i, j)$ from equation (2). $I($.$) is the indicator$ function, from equation (5).

We plot the channel capacity from equation (7), for an exemplary visual MIMO system, where the transmit elements of the LEA are light emitting diodes (LEDs) and the receiver is a machine vision camera (Basler Pilot piA640), over a range of distances $d$ (Fig. 5) and over different viewing angles $\phi$ (Fig. 6). The underlying parameters used in our analysis are summarized in Table I.

Inferences: From the analytical capacity plots we draw few notable inferences that relate to the multiplexing and diversity characterizations in visual MIMO.

- The visual MIMO system with no blur can achieve capacities of the order of Mbps even at long distances of about $90 \mathrm{~m}$. Blurring certainly reduces multiplexing range but still medium ranges of 30-40m are achievable at high data rates. The data rate gains at these distances are attributed to multiplexing where each LED sends an independent stream of bits over parallel channels. The transitions in the plot (for the multi LED cases) indicate the switch from multiplexing to diversity mode. The capacity gains due to diversity at the long distances, though may not be significant comparable to the multiplexing gains at shorter distances, are still close to an order of magnitude gain compared to the single LED system. 


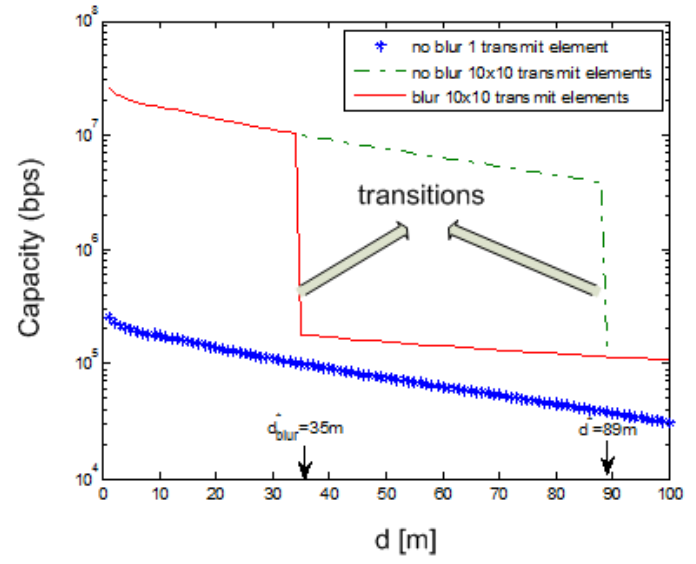

Fig. 5. Visual MIMO channel Capacity versus distance $(\phi=0)$

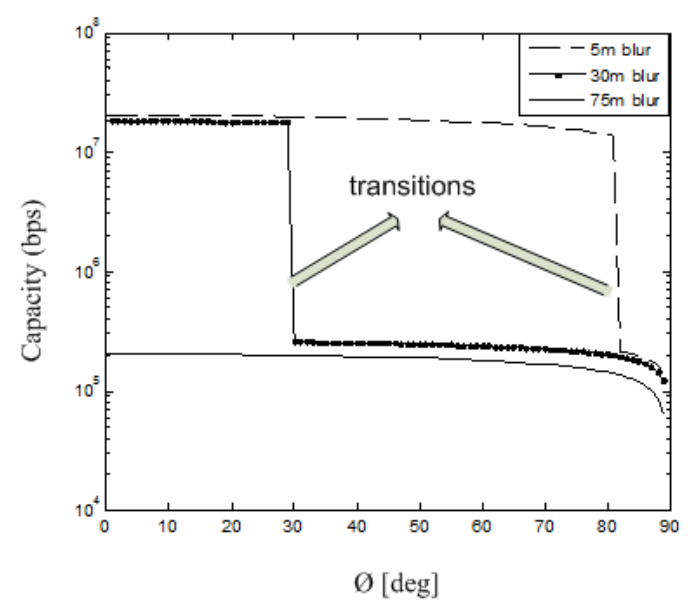

Fig. 6. Visual MIMO channel Capacity versus angle ( $d$ constant)

- A visual MIMO system will have to switch between the multiplexing and diversity modes in discrete intervals based on distance and angle unlike RF MIMO where the gains in these modes could be achieved simultaneously but follow a continuous trade-off in performance. Moreover, a visual MIMO system will have to switch autonomously between these modes depending on the orientation of the receiver with respect to the transmitter in order to leverage the gains. This suggests that the throughput of visual MIMO links can be significantly improved through rate adaptation techniques, which adapt the transmission scheme to the receiver perspective.

- In RF MIMO, in order to select the mode of operation (multiplexing and/or diversity) the channel state information (CSI) has to be known or estimated which either incurs a data overhead and/or complex receiver processing. But in visual MIMO, since the optical channel is deterministic in nature the overhead in selecting either of the modes of operations will be very less because the need to send preamble bits to determine the channel information (distance,angle etc.) may be obviated by the use of efficient computer vision techniques [6]. Since fading is negligible the complexity in estimating the CSI to exploit MIMO techniques is lesser than in RF but still leads to interesting challenges in computer vision and image processing.

- The visual MIMO channel capacity is consistent over a wide range of viewing angles (small or large depends on distance). We see that the system can achieve large multiplexing gains at short distances and at almost all viewing angles which implies that the system would be robust to any misalignment between the transmitter and receiver. Its cleat that at large distances (of the order of $75 \mathrm{~m}$ ), due to the effect of lens blur, the LEDs may not be resolved easily even at $\phi=0$ and hence at such distances where multiplexing will fail but using diversity over all angles can still offer an order of gain in data rates.

Such consistency in data rates over angular misalignment is important especially in mobile settings as the choice of multiplexing and/or diversity depends largely on the orientation of the mobile devices at each instance of time. This is in strong contrast to the RF systems (even MIMO) where the the signal can drop significantly with mobility especially when there is a deep fade in the channel or at high mobile velocities.

\begin{tabular}{|l|c|}
\hline Parameter & Value \\
\hline$P_{\text {in }}[\mathrm{mW}]$ & 100 \\
\hline$F O V \psi[\mathrm{deg}]$ & 42 \\
\hline$A_{\text {lens }}\left[\mathrm{mm}^{2}\right]$ & 15.7 \\
\hline$P_{n}\left[\mathrm{~mW} / \mathrm{cm}^{2}\right]$ & 600 \\
\hline$l[\mathrm{~mm}]$ & 6 \\
\hline$f[\mathrm{~mm}]$ & 8.5 \\
\hline$s[\mu]$ & 7.1 \\
\hline$\alpha[\mathrm{cm}]$ & 5 \\
\hline$\sigma_{\text {blur }}[\mu]$ & 0.5 \\
\hline $\mathrm{R}$ & 0.54 \\
\hline$\Phi[\mathrm{deg}]$ & 20 \\
\hline$\alpha[\mathrm{cm}]$ & 5 \\
\hline
\end{tabular}

TABLE I

TABLE OF PARAMETER VAlues BASLER Pilot PIA640 CAMERA $\left(\sigma_{b l u r}, f\right.$ ARE MEASURED USING CAMERA CALIBRATION EXPERIMENTS [1], [12])

\section{CONCLUSION}

We showed that visual MIMO can enable high data rate mobile optical wireless communication over long transmission ranges. This concept advocates that regardless of any type of modulation and transmission scheme, the system can achieve high data rates simply by exploiting some of the unique characteristics of the visual channel. The visual MIMO approach, different from that of its RF counterpart, allows adaptive design where multiplexing gains can be obtained at short distances while ranges of hundreds of meters can be achieved in a diversity mode. Our analytical results report even in the presence of signal distortion due to lens blur channel capacities of the order of Mbps at short distances and of the order of hundreds of Kbps at medium to longer ranges 
for an exemplary visual MIMO system with 100 LEDs in an array. We also showed similar channel capacities for the same system over wide camera view angles. These results validate the premise that the MIMO gains in an optical MIMO system such as visual MIMO is primarily dependent on receiver perspective with respect to the transmitter in contrast to the multipath fading dependent gains in RF MIMO. We inferred that a visual MIMO system will have to switch between its multiplexing and diversity mode unlike RF MIMO where they can be achieved simultaneously but follow a tradeoff in performance. The consistency in data rates over a wide range of camera viewing angles is a positive indication that visual MIMO can enable mobility in optical wireless communication.

\section{REFERENCES}

[1] Camera calibration toolbox for matlab. http://www.vision.caltech.edu/ bouguetj/calib_doc/index.html\#links.

[2] Free space optics:technology insight. www.freespaceoptics.org.

[3] Irda. http://http://www.irda.org/index.cfm/.

[4] Mipav. http://mipav.cit.nih.gov/documentation/HTML\%20Algorithms/ FiltersSpatialGaussianBlur.html.

[5] Stan moore astronomy. http://www.stanmooreastro.com/pixel size.html.

[6] A.Ashok, M.Gruteser, N. B. Mandayam, J. Silva, K. Dana, and M.Varga. Challenge: Mobile optical networks through visual mimo. In MobiCom '10: Proceedings of the sixteenth annual international conference on Mobile computing and networking, pages 105-112, New York, NY, USA, 2010. ACM.

[7] S. Arai, S. Mase, T. Yamazato, T. Endo, T. Fujii, M. Tanimoto, K. Kidono, Y. Kimura, and Y. Ninomiya. Experimental on hierarchical transmission scheme for visible light communication using led traffic light and high-speed camera. In Vehicular Technology Conference, 2007. VTC-2007 Fall. 2007 IEEE 66th, pages 2174-2178, 30 2007-Oct. 3 2007.

[8] H. Binti Che Wook, T. Komine, S. Haruyama, and M. Nakagawa. Visible light communication with led-based traffic lights using 2-dimensional image sensor. In Consumer Communications and Networking Conference, 2006. CCNC 2006. 3rd IEEE, volume 1, pages 243-247, Jan. 2006.

[9] S. Borman. Raytracing and the camera matrix - a connection. A tutorial on the relationships between raytracing formulations of projective geometry and the standard camera matrix representation, June 2003.

[10] G. J. Foschini and M. J. Gans. On limits of wireless communications in a fading environment when using multiple antennas. Wireless Personal Comunications: Kluwer Academic, 6(3):311-355, March 1998.

[11] A. Goldsmith. Wireless Communications. Cambridge, 2005.

[12] B. K P Horn. Robot vision. MIT Press, Cambridge, MA, USA, 1986.

[13] S. Hranilovic and F.R. Kschischang. A pixelated mimo wireless optical communication system. Selected Topics in Quantum Electronics, IEEE Journal of, 12(4):859 -874, jul. 2006.

[14] C.C. Sun I. Moreno. Modelling the radiation pattern of leds. Optics Express, 16(3):1808-1819, Feb 2008.

[15] S. Jivkova, B.A. Hristov, and M. Kavehrad. Power-efficient multispotdiffuse multiple-input-multiple-output approach to broad-band optical wireless communications. Vehicular Technology, IEEE Transactions on, 53(3):882 - 889, may. 2004.

[16] J.M. Kahn and J.R. Barry. Wireless infrared communications. Proceedings of the IEEE, 85(2):265-298, Feb 1997.

[17] T. Komine and M. Nakagawa. Integrated system of white led visiblelight communication and power-line communication. IEEE Transactions on Consumer Electronics, , 49(1):71-79, Feb. 2003.

[18] T. Komine and M. Nakagawa. Fundamental analysis for visible-light communication system using led lights. IEEE Transactions on Consumer Electronics, 50(1):100-107, Feb 2004.

[19] C. Liang, M. Garfield, and K. R Dandekar T. P. Kurzweg. Mimo spacetime coding for diffuse optical communication. Microwave and Optical Technology Letters, 48:1108 - 1110, may. 2006.

[20] S. Perli, N. Ahmed, and D. Katabi. Pixnet: Lcd-camera pairs as communication links. In SIGCOMM, pages 451-452, 2010.
[21] T. Saito, S. Haruyama, and M. Nakagawa. Inter-vehicle communication and ranging method using led rear lights. Proceedings of the fifth IASTED international conference on Communication Systems and Networks, 5:278-283, Aug 2006.

[22] H. Sugiyama, S. Haruyama, and M.Nakagawa. Experimental investigation of modulation methods for visible light communications. IEEE Transactions on Communications,, 89(12):3393-3400, Dec 2006.

[23] A.P. Tang, J.M. Kahn, and Keang-Po Ho. Wireless infrared communication links using multi-beam transmitters and imaging receivers. In Communications, 1996. ICC 96, Conference Record, Converging Technologies for Tomorrow's Applications. 1996 IEEE International Conference on, volume 1, pages 180-186 vol.1, Jun 1996.

[24] D. N. C. Tse, P. Vishwanath, and L. Zheng. Diversity-multiplexing tradeoff in multiple-access channels. IEEE Trans. Inform. Theory, 50(9):1859-1874, September 2004.

[25] H. Willebrand and B. Ghuman. Free Space Optics:Enabling Optical Connectivity in Today's Networks. Sams, 2002.

[26] L. Zeng, D. C O'Brien, H.L Minh, G. E. Faulkner, K. Lee, D. Jung, Y. Oh, and E. T Won. High data rate multiple input multiple output (mimo) optical wireless communications using white led lighting. IEEE J.Sel. A. Commun., 27(9):1654-1662, 2009.

[27] L. Zheng and D. N. C. Tse. Diversity and multiplexing: A fundamental tradeoff in multiple-antenna channels. IEEE Trans. Inform. Theory, 49(5):1073-1096, May 2003. 